\title{
Antineoplastic Hormonal/Endocrine Agent
}

National Cancer Institute

\section{Source}

National Cancer Institute. Antineoplastic Hormonal/Endocrine Agent. NCI Thesaurus.

Code $\mathrm{C129818.}$

Any agent that affects hormone levels and exerts antineoplastic effects. Antineoplastic hormonal/endocrine agents treat either hormone-dependent or hormone-sensitive cancers by modulating hormone levels and manipulating the endocrine system. 